\title{
Diagnóstico de capacidades de innovación desde la perspectiva de los modelos de madurez
}

\section{A maturity model-based diagnosis of innovation capability \\ Diagnóstico das capacidades de inovação desde a perspectiva dos modelos de maturidade}

\author{
José E. Arias-Pérez ${ }^{1}$, Nelson E. Lozada-Barahona', Geovanny Perdomo-Charry ${ }^{2}$ \\ 1 Adm, Esp, MSc Departamento de Ciencias Administrativas, Universidad de Antioquia. \\ 2 Adm, Esp, MSc, PhD. Departamento de Ciencias Administrativas, Universidad de San Buenaventura. \\ Email: joenriquearias@posgradoseconomicas.com
}

Recibido: agosto 21 de $2013 \quad$ Aceptado: abril 06 de 2016

\begin{abstract}
Resumen
En la ciudad de Medellín, Colombia, se encuentran algunas de las grandes empresas del país líderes en l+D+i, sin embargo, a la fecha son pocos los estudios realizados en función de las capacidades de innovación $(\mathrm{Cl})$ subyacentes a sus buenos resultados en esta materia. Por ello, el objetivo del presente artículo es establecer la madurez de las capacidades de innovación de cinco empresas líderes en I+D+i, pertenecientes a cinco sectores claves de la industria: Licores, Cerámica Sanitaria, Electro-gasodomésticos, Cárnicos, y Concentrados para Animales. En cuanto a la metodología, la investigación es cuantitativa de corte transversal; para procesar los datos, se construyó un índice de Cl y se calcularon los rangos de los tres niveles de madurez por variables, lo cual permitió clasificar a las empresas objeto de estudio. En cuanto a los resultados, se encontró que sólo tres $\mathrm{Cl}$ se encuentran en el nivel de madurez Integración, Sinergia y Autonomía en las cinco empresas: Identificación de puntos de decisión claves en el proceso de los proyectos de innovación; Valores y políticas organizacionales; y Conformación de equipos de innovación. En conclusión, las $\mathrm{Cl}$ del área clave Proceso de Innovación, presentan los mayores niveles de madurez, en comparación con las de Conocimiento y Competencia, y Soporte Organizacional; lo cual evidencia que las compañías están más interesadas en las $\mathrm{Cl}$ duras, de corte tecnológico, que en las blandas de corte cultural o estratégico.
\end{abstract}

Palabras claves: capacidades de innovación, gestión de innovación, gestión tecnológica, madurez de capacidades de innovación, modelos de madurez

\begin{abstract}
Some of Colombia's largest leading R\&D+I companies are located in the city of Medellin; however, few studies have been carried out to date regarding the innovation capability (IC) underlying their good results in this area. This article has thus been aimed at establishing the IC maturity of five leading R\&D+I companies from five key industrial sectors: alcoholic beverages, sanitary ceramics, electric-gas domestic appliances, meat products and animal feed concentrates. A quantitative cross-sectional approach was adopted; an IC index was constructed for processing the data and the ranges of the three levels of maturity were calculated by variables, thereby enabling the companies to be classified. The results showed that only three IC came within integration, synergy and autonomy maturity levels in the five companies: identifying key
\end{abstract}


decision-making points concerning innovation projects, organisational values and policies and forming innovation teams. The key area 'innovation process' had the greatest IC maturity levels compared to those for knowledge and competence and organisational support, showing that companies were more interested in technology-based hard IC, than in soft cultural or strategic IC.

Key words: innovation capability, innovation management, technology management, innovation capability and maturity model, maturity model.

\section{Resumo}

Na cidade de Medellin, na Colômbia, estão algumas das grandes empresas líderes do país em R+D+i, no entanto, até o momento poucos estudos tem se feito sobre a capacidade de inovação $(\mathrm{Cl})$ que estão detrás dos bons resultados nesta área. Portanto, o objetivo deste trabalho é estabelecer a maturidade das capacidades de inovação das cinco companhias líderes em $\mathrm{R}+\mathrm{D}+\mathrm{i}$, pertencentes a cinco setores-chave da indústria: Licores, Cerâmica Sanitária, aparelhos eletro-gás, carne e Concentrados para animais. Em termos da metodologia, a pesquisa é quantitativa, transversal, para processar os dados, foi feito um índice de $\mathrm{Cl}$ e intervalos calculados de três níveis de maturidade para as variáveis, o que permitiu classificar as empresas em estudo. Quanto aos resultados, verificou-se que apenas três Cl estão no nível de maturidade integração, sinergia e autonomia em cinco empresas: Identificação dos principais pontos de decisão no processo de projetos de inovação; Valores e políticas organizacionais e integrar equipes de inovação. Em conclusão, as $\mathrm{Cl}$ da área-chave Processo de Inovação, têm os mais altos níveis de maturidade, em comparação com aquelas de Conhecimento e Competência, e Suporte Organizacional, o que mostra que as empresas estão mais interessadas nas Cl duras, que tem relação com a tecnologia, que nas áreas que tem relação com aspectos culturais ou estratégicos.

Palavras chave: capacidade de inovação, gestão da inovação, gestão da tecnologia, maturidade das capacidades de inovação, modelos de maturidade.

\section{Introducción}

En la ciudad de Medellín se encuentran algunas de las empresas de la industria colombiana reconocidas por su liderazgo en innovación (Botero y Castaño, 2006), el cual se ha venido consolidando por la puesta en marcha de distintas estrategias como la creación de centros de I+D, las alianzas con las universidades, el dinamismo en la adquisición de tecnología, la implementación de mecanismos de captura de nuevas ideas procedentes de los clientes y empleados, entre otras.

De tal suerte que la innovación está dejando de ser un hecho accidental, para convertirse en algo que actualmente se gestiona, sistematiza, mide y controla; por ello, estas empresas con el ánimo de aumentar la efectividad en esta materia, han venido interviniendo las diversas variables organizacionales, buscando transformarlas y alinearlas con ese propósito; lo que en otras palabras equivale a desarrollar capacidades de innovación $(\mathrm{Cl})$.

Sin embargo, en el ámbito local son pocos los ejercicios académicos que se han realizado con el propósito de establecer en qué estado se encuentran las $\mathrm{Cl}$, de ellos, los más destacados suelen enfocarse en el aspecto tecnológico en detrimento del cultural, y se han realizado en sectores específicos como el eléctrico (Robledo et.al. 2010) y de desarrollo de Software
(Aguirre, 2010) donde los resultados en innovación no son los más destacados.

La ausencia de estos ejercicios dificulta tanto la identificación de las fortalezas y los aspectos a mejorar en esta materia, como la transferencia de buenas prácticas de las empresas líderes a las seguidoras y novatas que desconocen cuál es el estándar en gestión de la innovación validado en el ámbito local.

De ahí la importancia de este artículo, que busca establecer la madurez de $\mathrm{Cl}$ en cinco empresas líderes en $++D+i$ en sus respectivos sectores industriales: Embutidos, Licores, Cerámica Sanitaria, Electro-gasodomésticos, y Concentrados para animales. Para este propósito, se utiliza el modelo desarrollado por Essmann y Du Preez (2009), que integra las dos perspectivas más importantes en esta materia, la tecnológica y la estratégica, el cual se compone de cuarenta y dos variables ampliamente mencionadas en la literatura, agrupadas en tres áreas claves: Proceso de Innovación, Conocimiento y Competencia, y Soporte Organizacional. Sumado a ello, incluye una escala de madurez con tres niveles: Ad hoc y Limitado, Formalizado y Predecible, e Integración, Sinergia y Autonomía.

En cuanto al procesamiento de los datos se recurre fundamentalmente a la metodología desarrollada por Jung et al., (2009), quienes sugieren el análisis cluster 
para determinar los rangos de los niveles de la escala de madurez, si bien su aplicación inicial se realizó en función de otro constructo teórico, puede extrapolarse a los modelos de madurez de $\mathrm{Cl}$ que tradicionalmente tienen debilidades en esta materia (López 2009, Robledo et al,. 2010).

\section{Materiales y métodos}

La investigación es cuantitativa de corte transversal, dado que la recolección de datos se realiza en un solo momento en el tiempo, los cuales permiten determinar los valores en los que se manifiestan las cuarenta y dos $\mathrm{Cl}$ (Hernández et al.,2004).

Las empresas seleccionadas para el estudio, son reconocidas por su liderazgo en $\mathrm{I}+\mathrm{D}+\mathrm{i}$, en cinco sectores claves de la industria local, a saber, Licores, Cerámica Sanitaria, Electro-gasodomésticos, Embutidos, y Concentrados para Animales. Incluso se les reconoce la profundidad de la relación con las universidades líderes en investigación de la región, expresada en un creciente número de proyectos conjuntos. Las personas encuestadas pertenecen a los Departamentos de Investigación, Desarrollo e Innovación ( $1+\mathrm{D}+\mathrm{i})$ de las respectivas compañías, quienes se caracterizan por tener una amplia visión de las distintas prácticas de innovación que han sido implementadas.

Para la medición de las $\mathrm{Cl}$, se utilizó el instrumento propuesto por Essman (2009), quien plantea utilizar el modelo de madurez como escala de medición, con sus tres niveles Ad hoc y Limitado, Formalizado y Predecible, e Integración, Sinergia y Autonomía.

Para el cálculo de la madurez de las $\mathrm{Cl}$, se realizaron los siguientes cuatro pasos: el primero, corresponde a la relativización de las variables; el segundo a la construcción de un índice de $\mathrm{Cl}$ por empresas (Lema, 2002); el tercero al establecimiento de los rangos de los tres niveles de madurez para cada una de las cuarenta y dos variables (Jung et al., 2009) y el cuarto a la clasificación de las empresas, ubicando su respectivo índice en los rangos.

Con respecto a la relativización de las variables, necesaria para la realización de análisis posteriores como el Cluster, se procedió a asignar un peso a cada una de ellas en un panel de expertos, el cual se multiplicó por la calificación de las mismas y se dividió por la sumatoria de todas las ponderaciones al interior de las tres áreas claves (Lema, 2002).

El segundo paso, corresponde a la construcción del Índice de Valor de Importancia (IVI), que corresponde al promedio de las calificaciones de las cuarenta y dos variables en cada una de las cinco empresas (Tabla 1).

En cuanto al establecimiento de los rangos de los tres niveles de la escala de madurez (Figura 1), se realizó un análisis Cluster, en el cual se aplicó la técnica no jerárquica K-means, que se usa especialmente cuando se conoce de antemano el número de grupos o conglomerados (Jung et al., 2009).

En este caso, se tomó la decisión de agrupar las observaciones en dos conglomerados, con el propósito de contar con dos números ubicados en una escala de 0 a 5, con los cuales se construyó el primer rango que sirve de base para determinar los otros dos, a partir de los valores que están por debajo de su límite inferior o por encima del superior.

Luego, se realizó una sumatoria de los valores que arroja el análisis cluster de las variables de cada área clave, tanto los que pertenecen al conglomerado 1 como al 2, y se construyó el primer rango correspondiente al nivel Formalizado y Predecible, identificando el límite superior e inferior, luego se elaboraron los otros dos de la forma descrita anteriormente (Figura 1). En este punto es posible determinar la madurez de las empresas, al ubicar el IVI por variables de cada empresa en el respectivo rango (Tabla 1 ).

\section{Resultados}

El análisis de los resultados se realiza por cada una de las tres áreas claves y sus respectivas variables, buscando identificar en cuál de las tres categorías de la escala de madurez: Ad Hoc y Limitado, Formalizado y Predecible, e Integración, Sinergia y Autonomía, se encuentran las cinco empresas objeto del estudio.

\section{Capacidad de Innovación del Área Clave Proceso de Innovación}

Al revisar los datos se pudo observar que en la escala de madurez Ad Hoc y Limitado solo una empresa, la que corresponde al sector de los Concentrados para Animales, ubica tres de las variables: Vinculación de clientes y proveedores a los procesos de innovación; Planeación y coordinación de los proyectos de innovación; y Utilización de técnicas de gestión de proyectos. Las otras empresas localizan todas sus variables en las escalas de madurez Formalizado y Predecible; e Integración, Sinergia y Autonomía (Figura 1).

También es importante anotar que la empresa del sector Cárnico responde a las variables en su gran mayoría ubicándolas en la escala de madurez Integración 


\begin{tabular}{|c|c|c|c|}
\hline $\mathrm{CI}$ del área clave proceso de innovación & $\begin{array}{l}\text { Ad Hoc y } \\
\text { limitado }\end{array}$ & $\begin{array}{l}\text { Formalizado y } \\
\text { predecible }\end{array}$ & $\begin{array}{c}\text { Integración, } \\
\text { sinergia y } \\
\text { autonomía }\end{array}$ \\
\hline 1. Comprensión del mercado. & & & \\
\hline $\begin{array}{l}\text { 2. Vinculación de clientes y proveedores a los procesos } \\
\text { de innovación. }\end{array}$ & - $\bullet$ & & \\
\hline 3. Identificación de oportunidades e ideas. & & & \\
\hline 4. Desarrollo de conceptos. & & & \\
\hline 5. Exploración y detección de oportunidades latentes. & & & \\
\hline 6. Contextualización de las oportunidades y conceptos. & & & \\
\hline $\begin{array}{l}\text { 7. Examen, depuración y priorización de las oportunidades } \\
\text { y conceptos. }\end{array}$ & & & \\
\hline 8. Desarrollo, implementación y explotación de los concept & & & \\
\hline 9. Planeación y coordinación de los proyectos de innovació & & & \\
\hline 10. Asignación de los recursos a la innovación. & & & \\
\hline 11. Balance del portafolio de proyectos de innovación. & & & \\
\hline 12. Utilización de técnicas de gestión de proyectos. & & & \\
\hline $\begin{array}{l}\text { 13. Identificación de puntos de decisión claves en el proces } \\
\text { proyectos de innovación. }\end{array}$ & & & \\
\hline 14. Principios para orientar el proceso de toma de decisione & & & \\
\hline 15. Reducción de la incertidumbre y mitigación del riesgo. & & & \\
\hline
\end{tabular}

Empresa sector cárnico

Empresa sector cerámico

- Empresa sector electro-gasodomésticos

.... Empresa sector concentrados para animales

Empresa sector licores

Fuente: elaboración propia.

Figura 1. Madurez del área clave proceso de innovación por empresas 
Tabla 1. Índice de valor de importancia de las empresas por capacidades de innovación

\begin{tabular}{|c|c|c|c|c|c|c|c|c|}
\hline \multirow[b]{2}{*}{ Capacidades de innovación } & \multicolumn{5}{|c|}{ IVI de las empresas } & \multicolumn{3}{|c|}{ Rangos escala de madurez } \\
\hline & $\begin{array}{l}\text { Sector } \\
\text { cár- } \\
\text { nico }\end{array}$ & $\begin{array}{l}\text { Sector } \\
\text { cerá- } \\
\text { mico }\end{array}$ & $\begin{array}{l}\text { Sector } \\
\text { electro y } \\
\text { gasodo- } \\
\text { més- } \\
\text { ticos }\end{array}$ & $\begin{array}{l}\text { Sector } \\
\text { concentra- } \\
\text { dos para } \\
\text { animales }\end{array}$ & $\begin{array}{l}\text { Sector } \\
\text { licores }\end{array}$ & $\begin{array}{l}\text { Ad hoc } \\
\text { y limi- } \\
\text { tado }\end{array}$ & $\begin{array}{l}\text { Formaliza- } \\
\text { do y prede- } \\
\text { cible }\end{array}$ & $\begin{array}{l}\text { Integra- } \\
\text { ción, } \\
\text { siner- } \\
\text { gia y auto- } \\
\text { nomía }\end{array}$ \\
\hline 1. Comprensión del Mercado. & 0,32 & 0,25 & 0,33 & 0,23 & 0,24 & $0 \geq \mathrm{n}<0,19$ & $0,19 \geq n<0,3$ & $\mathrm{n} \geq 0,3$ \\
\hline $\begin{array}{l}\text { 2. Vinculación de clientes y proveedores a } \\
\text { los procesos de innovación. }\end{array}$ & 0,12 & 0,09 & 0,09 & 0,05 & 0,13 & $0 \geq \mathrm{n}<0,08$ & $0,08 \geq n<0,12$ & $\mathrm{n} \geq 0,12$ \\
\hline 3. Identificación de oportunidades e ideas. & 0,38 & 0,38 & 0,26 & 0,35 & 0,36 & $0 \geq \mathrm{n}<0,21$ & $0,21 \geq n<0,39$ & $\mathrm{n} \geq 0,39$ \\
\hline 4. Desarrollo de conceptos. & 0,47 & 0,38 & 0,43 & 0,31 & 0,43 & $0 \geq \mathrm{n}<0,26$ & $0,26 \geq \mathrm{n}<0,47$ & $\mathrm{n} \geq 0,47$ \\
\hline $\begin{array}{l}\text { 5. Exploración y detección de } \\
\text { oportunidades latentes. }\end{array}$ & 0,28 & 0,26 & 0,23 & 0,23 & 0,25 & $0 \geq \mathrm{n}<0,16$ & $0,16 \geq n<0,28$ & $\mathrm{n} \geq 0,28$ \\
\hline $\begin{array}{l}\text { 6. Contextualización de las oportunidades } \\
\text { y conceptos. }\end{array}$ & 0,43 & 0,36 & 0,43 & 0,33 & 0,42 & $0 \geq n<0,28$ & $0,28 \geq n<0,45$ & $\mathrm{n} \geq 0,45$ \\
\hline $\begin{array}{l}\text { 7. Examen, depuración y priorización de las } \\
\text { oportunidades y conceptos. }\end{array}$ & 0,30 & 0,24 & 0,26 & 0,28 & 0,32 & $0 \geq \mathrm{n}<0,18$ & $0,18 \geq n<0,33$ & $\mathrm{n} \geq 0,33$ \\
\hline $\begin{array}{l}\text { 8. Desarrollo, implementación y explotación } \\
\text { de los conceptos. }\end{array}$ & 0,44 & 0,37 & 0,42 & 0,29 & 0,35 & $0 \geq \mathrm{n}<0,27$ & $0,27 \geq n<0,41$ & $\mathrm{n} \geq 0,41$ \\
\hline $\begin{array}{l}\text { 9. Planeación y coordinación de los } \\
\text { proyectos de innovación. }\end{array}$ & 0,31 & 0,24 & 0,26 & 0,17 & 0,27 & $0 \geq \mathrm{n}<0,19$ & $0,19 \geq n<0,29$ & $\mathrm{n} \geq 0,29$ \\
\hline $\begin{array}{l}\text { 10. Asignación de los recursos a la } \\
\text { innovación. }\end{array}$ & 0,23 & 0,15 & 0,19 & 0,18 & 0,18 & $0 \geq n<0,15$ & $0,15 \geq n<0,2$ & $\mathrm{n} \geq 0,2$ \\
\hline $\begin{array}{l}\text { 11. Balance del portafolio de proyectos de } \\
\text { innovación. }\end{array}$ & 0,21 & 0,15 & 0,16 & 0,15 & 0,17 & $0 \geq \mathrm{n}<0,11$ & $0,11 \geq n<0,16$ & $\mathrm{n} \geq 0,16$ \\
\hline $\begin{array}{l}\text { 12. Utilización de técnicas de gestión de } \\
\text { proyectos. }\end{array}$ & 0,14 & 0,14 & 0,18 & 0,06 & 0,15 & $0 \geq \mathrm{n}<0,11$ & $0,11 \geq n<0,16$ & $\mathrm{n} \geq 0,16$ \\
\hline $\begin{array}{l}\text { 13. Identificación de puntos de decisión } \\
\text { claves en el proceso de los proyectos de } \\
\text { innovación. }\end{array}$ & 0,03 & 0,03 & 0,03 & 0,03 & 0,03 & $0 \geq n<0,02$ & $0,02 \geq n<0,03$ & $\mathrm{n} \geq 0,03$ \\
\hline $\begin{array}{l}\text { 14. Principios para orientar el proceso de } \\
\text { toma de decisiones. }\end{array}$ & 0,06 & 0,06 & 0,05 & 0,06 & 0,06 & $0 \geq \mathrm{n}<0,05$ & $0,05 \geq n<0,06$ & $\mathrm{n} \geq 0,06$ \\
\hline $\begin{array}{l}\text { 15. Reducción de la incertidumbre y } \\
\text { mitigación del riesgo. }\end{array}$ & 0,09 & 0,07 & 0,07 & 0,10 & 0,07 & $0 \geq \mathrm{n}<0,04$ & $0,04 \geq n<0,09$ & $\mathrm{n} \geq 0,09$ \\
\hline $\begin{array}{l}\text { 16. Estrategia de desarrollo y/o adquisición de } \\
\text { tecnología y conocimiento. }\end{array}$ & 0,40 & 0,29 & 0,43 & 0,23 & 0,38 & $0 \geq \mathrm{n}<0,28$ & $0,28 \geq n<0,46$ & $\mathrm{n} \geq 0,46$ \\
\hline 17. Direccionamiento de la investigación. & 0,26 & 0,25 & 0,22 & 0,13 & 0,23 & $0 \geq \mathrm{n}<0,20$ & $0,20 \geq \mathrm{n}<0,27$ & $\mathrm{n} \geq 0,27$ \\
\hline $\begin{array}{l}\text { 18. Identificación y extracción de información } \\
\text { relevante. }\end{array}$ & 0,46 & 0,32 & 0,33 & 0,45 & 0,36 & $0 \geq n<0,29$ & $0,29 \geq n<0,45$ & $\mathrm{n} \geq 0,45$ \\
\hline $\begin{array}{l}\text { 19. Captura, almacenamiento y acceso a los } \\
\text { datos y la información. }\end{array}$ & 0,40 & 0,26 & 0,34 & 0,15 & 0,34 & $0 \geq n<0,27$ & $0,27 \geq n<0,39$ & $\mathrm{n} \geq 0,39$ \\
\hline $\begin{array}{l}\text { 20. Gestión de las competencias y las } \\
\text { tecnologías centrales. }\end{array}$ & 0,20 & 0,15 & 0,14 & 0,08 & 0,20 & $0 \geq n<0,13$ & $0,13 \geq n<0,21$ & $\mathrm{n} \geq 0,21$ \\
\hline $\begin{array}{l}\text { 21. Desarrollo y adquisición de competencias } \\
\text { y tecnologías centrales. }\end{array}$ & 0,52 & 0,44 & 0,40 & 0,31 & 0,58 & $0 \geq \mathrm{n}<0,34$ & $0,34 \geq n<0,66$ & $\mathrm{n} \geq 0,66$ \\
\hline $\begin{array}{l}\text { 22. Definición de la política de propiedad } \\
\text { intelectual. }\end{array}$ & 0,10 & 0,06 & 0,09 & 0,06 & 0,09 & $0 \geq \mathrm{n}<0,24$ & $0,24 \geq n<0,33$ & $\mathrm{n} \geq 0,33$ \\
\hline 23. Gestión de la propiedad intelectual. & 0,33 & 0,18 & 0,28 & 0,27 & 0,31 & $0 \geq \mathrm{n}<0,24$ & $0,24 \geq n<0,33$ & $\mathrm{n} \geq 0,33$ \\
\hline
\end{tabular}




\begin{tabular}{|c|c|c|c|c|c|c|c|c|}
\hline \multirow[b]{2}{*}{ Capacidades de innovación } & \multicolumn{5}{|c|}{ IVI de las empresas } & \multicolumn{3}{|c|}{ Rangos escala de madurez } \\
\hline & $\begin{array}{l}\text { Sector } \\
\text { cár- } \\
\text { nico }\end{array}$ & $\begin{array}{l}\text { Sector } \\
\text { cerá- } \\
\text { mico }\end{array}$ & $\begin{array}{l}\text { Sector } \\
\text { electro y } \\
\text { gasodo- } \\
\text { més- } \\
\text { ticos }\end{array}$ & $\begin{array}{l}\text { Sector } \\
\text { concentra- } \\
\text { dos para } \\
\text { animales }\end{array}$ & $\begin{array}{l}\text { Sector } \\
\text { licores }\end{array}$ & $\begin{array}{l}\text { Ad hoc } \\
\text { y limi- } \\
\text { tado }\end{array}$ & $\begin{array}{l}\text { Formaliza- } \\
\text { do y prede- } \\
\text { cible }\end{array}$ & $\begin{array}{l}\text { Integra- } \\
\text { ción, } \\
\text { siner- } \\
\text { gia y auto- } \\
\text { nomía }\end{array}$ \\
\hline 25. Gestión del conocimiento tácito & 0,30 & 0,19 & 0,21 & 0,19 & 0,23 & $0 \geq \mathrm{n}<0,17$ & $0,17 \geq n<0,29$ & $\mathrm{n} \geq 0,29$ \\
\hline 26. Colaboración y networking interno. & 0,13 & 0,11 & 0,11 & 0,10 & 0,12 & $0 \geq \mathrm{n}<0,08$ & $0,08 \geq \mathrm{n}<0,15$ & $\mathrm{n} \geq 0,15$ \\
\hline 27. Colaboración y networking externo. & 0,46 & 0,42 & 0,44 & 0,35 & 0,54 & $0 \geq \mathrm{n}<0,38$ & $0,38 \geq \mathrm{n}<0,58$ & $\mathrm{n} \geq 0,58$ \\
\hline $\begin{array}{l}\text { 28. Formulación y comunicación de la } \\
\text { estrategia de innovación. }\end{array}$ & 0,39 & 0,22 & 0,26 & 0,30 & 0,37 & $0 \geq \mathrm{n}<0,28$ & $0,28 \geq \mathrm{n}<0,46$ & $\mathrm{n} \geq 0,46$ \\
\hline 29. Valores y políticas organizacionales. & 0,47 & 0,38 & 0,34 & 0,44 & 0,49 & $0 \geq n<0,20$ & $0,20 \geq \mathrm{n}<0,27$ & $\mathrm{n} \geq 0,27$ \\
\hline $\begin{array}{l}\text { 30. Prácticas y procedimientos } \\
\text { organizacionales. }\end{array}$ & 0,16 & 0,13 & 0,14 & 0,10 & 0,16 & $0 \geq n<0,29$ & $0,29 \geq \mathrm{n}<0,45$ & $\mathrm{n} \geq 0,45$ \\
\hline $\begin{array}{l}\text { 31. Fomento y apoyo al comportamiento } \\
\text { innovador. }\end{array}$ & 0,44 & 0,25 & 0,35 & 0,47 & 0,40 & $0 \geq \mathrm{n}<0,27$ & $0,27 \geq n<0,39$ & $\mathrm{n} \geq 0,39$ \\
\hline 32. Inversión en Innovación. & 0,20 & 0,13 & 0,14 & 0,13 & 0,16 & $0 \geq \mathrm{n}<0,13$ & $0,13 \geq n<0,21$ & $\mathrm{n} \geq 0,21$ \\
\hline $\begin{array}{l}\text { 33. Disponibilidad de recursos para la } \\
\text { innovación. }\end{array}$ & 0,38 & 0,21 & 0,28 & 0,23 & 0,27 & $0 \geq n<0,34$ & $0,34 \geq n<0,66$ & $\mathrm{n} \geq 0,66$ \\
\hline $\begin{array}{l}\text { 34. Infraestructura, sistemas y herramientas de } \\
\text { apoyo a la innovación. }\end{array}$ & 0,30 & 0,21 & 0,26 & 0,17 & 0,25 & $0 \geq \mathrm{n}<0,24$ & $0,24 \geq n<0,33$ & $\mathrm{n} \geq 0,33$ \\
\hline $\begin{array}{l}\text { 35. Estructura organizacional e infraestructura } \\
\text { instalada. }\end{array}$ & 0,21 & 0,20 & 0,21 & 0,19 & 0,19 & $0 \geq \mathrm{n}<0,24$ & $0,24 \geq \mathrm{n}<0,33$ & $\mathrm{n} \geq 0,33$ \\
\hline 36. Conformación de equipos de innovación. & 0,37 & 0,34 & 0,34 & 0,33 & 0,39 & $0 \geq \mathrm{n}<0,03$ & $0,03 \geq n<0,06$ & $\mathrm{n} \geq 0,06$ \\
\hline 37. Comunicación y flujo de la información. & 0,03 & 0,02 & 0,03 & 0,02 & 0,03 & $0 \geq \mathrm{n}<0,17$ & $0,17 \geq n<0,29$ & $\mathrm{n} \geq 0,29$ \\
\hline 38. Motivación de los individuos y equipos. & 0,22 & 0,14 & 0,20 & 0,20 & 0,21 & $0 \geq \mathrm{n}<0,08$ & $0,08 \geq \mathrm{n}<0,15$ & $\mathrm{n} \geq 0,15$ \\
\hline $\begin{array}{l}\text { 39. Contratación de nuevas personas y } \\
\text { asignación de roles. }\end{array}$ & 0,05 & 0,04 & 0,05 & 0,05 & 0,06 & $0 \geq \mathrm{n}<0,38$ & $0,38 \geq \mathrm{n}<0,58$ & $\mathrm{n} \geq 0,58$ \\
\hline 40. Medición de la innovación. & 0,21 & 0,13 & 0,18 & 0,18 & 0,17 & $0 \geq \mathrm{n}<0,17$ & $0,17 \geq \mathrm{n}<0,29$ & $\mathrm{n} \geq 0,29$ \\
\hline $\begin{array}{l}\text { 41. Implementación y mejora del modelo de } \\
\text { innovación. }\end{array}$ & 0,09 & 0,06 & 0,07 & 0,10 & 0,08 & $0 \geq \mathrm{n}<0,08$ & $0,08 \geq \mathrm{n}<0,15$ & $\mathrm{n} \geq 0,15$ \\
\hline 42. Benchmarking de la innovación & 0,11 & 0,09 & 0,12 & 0,10 & 0,08 & $0 \geq \mathrm{n}<0,38$ & $0,38 \geq n<0,58$ & $\mathrm{n} \geq 0,58$ \\
\hline
\end{tabular}

Fuente: elaboración propia.

Sinergia y Autonomía; de las quince variables que componen el área clave solo cinco de ellas, las localiza en la escala de madurez Formalizado y Predecible, son ellas: Vinculación de clientes y proveedores a los procesos de innovación; Identificación de oportunidades e ideas; Contextualización de las oportunidades y conceptos; Examen, depuración y priorización de las oportunidades y conceptos; y Utilización de técnicas de gestión de proyectos (Figura 1).

Las otras tres empresas, la que se desempeña en el sector Cerámico, la del sector Electro Gasodoméstico y la del sector de Licores, concentran su tendencia de $\mathrm{Cl}$ en la escala de madurez Formalizado y Predecible (Figura 1).
Las cinco empresas coinciden al ubicar tanto Contextualización de las oportunidades y conceptos, como Examen, depuración y priorización de las oportunidades y conceptos, en la escala de madurez Formalizado y Predecible; así mismo la variable Identificación de puntos de decisión claves en el proceso de los proyectos de innovación, está ubicada en la escala de Integración Sinergia y Autonomía para las cinco empresas estudiadas (Figura 1).

Capacidad de Innovación del Área Clave Conocimiento y Competencia

La variable Estrategia de desarrollo y/o adquisición de tecnología y conocimiento, se ubica en la escala de 
madurez Formalizado y Predecible para cuatro de las cinco empresas, solo la empresa del sector de Concentrados para Animales la localiza en la escala de madurez Ad Hoc y Limitado. Del mismo modo ocurre para las variables Gestión de las competencias y las tecnologías centrales; Desarrollo y adquisición de competencias y tecnologías centrales; Colaboración y networking externo. De lo anterior se puede observar que la empresa del sector de Concentrados para Animales tiende a concentrar su $\mathrm{Cl}$ en la escala de madurez Ad Hoc y Limitado, dado que de las doce variables que componen el área clave, ocho están ubicadas en esta área clave (Figura 2).
Existe coincidencia en las cinco empresas analizadas al ubicarse en la escala de madurez Ad Hoc y Limitado para la variable Definición de la política de propiedad intelectual; también hay coincidencia en la variable Colaboración y networking interno, pero en esta ocasión la escala de madurez en la que se localizan es Formalizado y Predecible (Figura 2).

Se observa que la empresa del sector de Licores ubica en su gran mayoría su nivel de $\mathrm{Cl}$ para el área clave en cuestión, en la escala de madurez Formalizado y Predecible, puesto que solo una variable de las doce, la denominada Definición de la política de propiedad

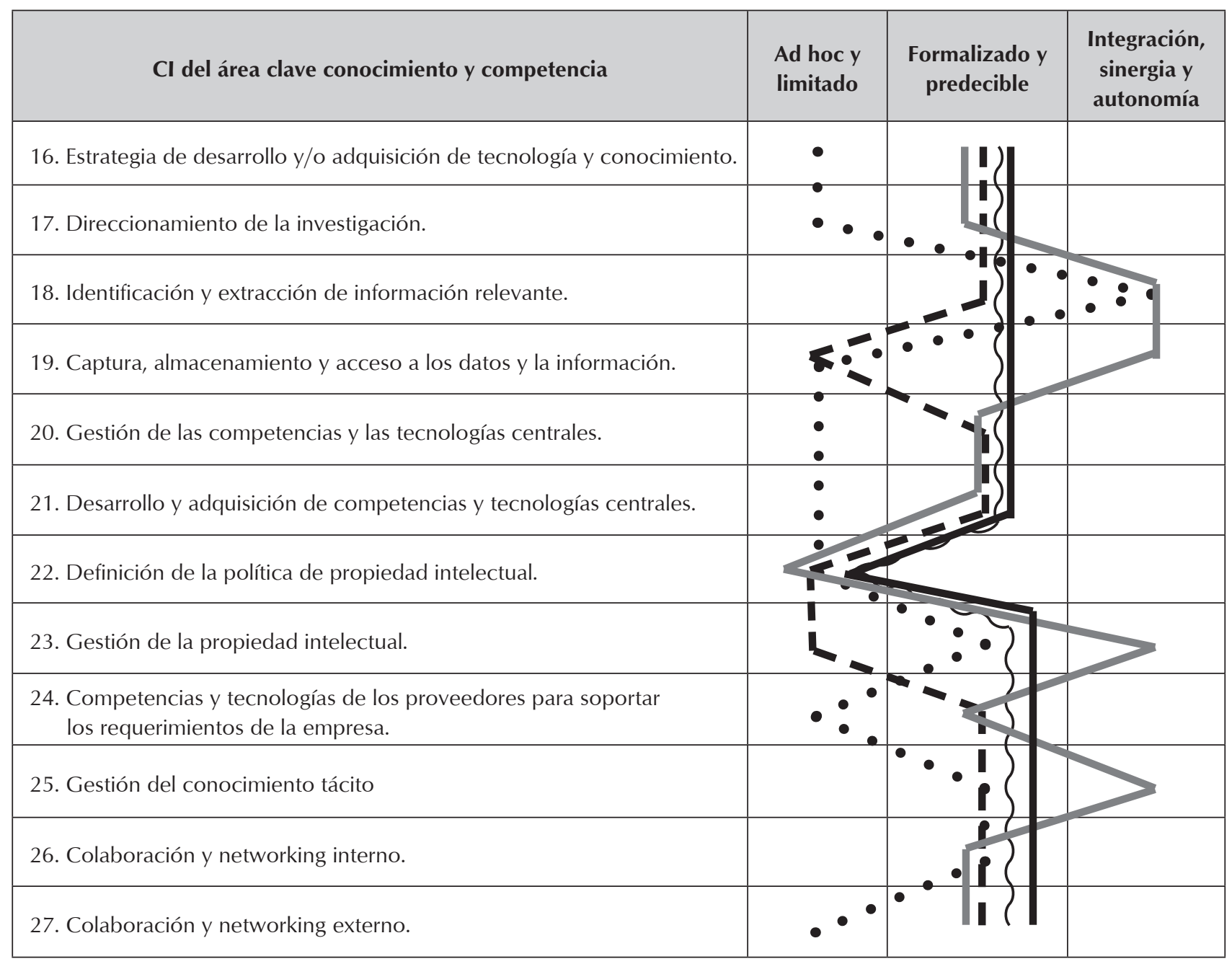

\begin{tabular}{|c|c|}
\hline$\widetilde{r}$ & $\begin{array}{l}\text { Empresa Sector Cárnico. } \\
\text { Empresa Sector Cerámico } \\
\text { Empresa Sector Electro-Gasodomésticos } \\
\text { Empresa Sector Concentrados para Animales } \\
\text { Empresa Sector Licores }\end{array}$ \\
\hline & Elaboración propia \\
\hline
\end{tabular}

Figura 2. Madurez del área clave conocimiento y competencia por empresas 
intelectual, se localizó en la escala de madurez Ad Hoc y Limitado. Por otro lado la empresa que presenta una mejor tendencia en el nivel de madurez es la del sector Cárnico, dado que logra localizar cuatro de las doce variables en la escala de madurez Integración, Sinergia y Autonomía, a saber, Identificación y extracción de información relevante, Captura, almacenamiento y acceso a los datos y la información, Gestión de la propiedad intelectual; Gestión del conocimiento tácito (Figura 2).

\section{Capacidad de Innovación del Área Clave Soporte Organizacional}

De las quince variables que componen esta área clave, en dos de ellas coinciden las cinco empresas estudiadas al ubicarse en la escala de madurez Integración Sinergia y Autonomía, son ellas: Valores y políticas organizacionales, y Conformación de equipos de innovación. También hay coincidencia en cinco de las doce, pero en esta ocasión en la escala de madurez Ad Hoc y Limitado, de las variables Prácticas y procedimientos organizacionales, Estructura organizacional e infraestructura instalada, Comunicación y flujo de la información, Contratación de nuevas personas y asignación de roles, e Implementación y mejora del modelo de innovación. Solo existe una coincidencia de las cinco empresas en la escala de madurez Formalizado y Predecible y es en la variable Inversión en Innovación (Figura 3).

Como se ha podido observar en los resultados de esta área clave, existe un alto grado de similitud en la $\mathrm{Cl}$ de las empresas estudiadas, dado que en ocho de las quince variables hay coincidencia; es importante mencionar que dicha similitud se concentra en dos escalas de madurez, la Ad Hoc y Limitado; e Integración, Sinergia y Autonomía (Figura 3).

\section{Discusión}

Un elemento que es importante tener en consideración según los resultados obtenidos en las cinco empresas estudiadas, es que de las tres áreas claves que componen el constructo de la capacidad de innovación, solo el área clave Proceso de Innovación presenta una madurez de la $\mathrm{Cl}$ que tiende a ubicarse en los dos niveles superiores (Formalizado y Predecible; e Integración, Sinergia y Autonomía); las otras dos áreas claves a saber, Conocimiento y Competencia; y Soporte Organizacional, observan niveles del madurez muy dispersos en las tres escalas consideradas en el estudio, incluso con una fuerte tendencia para localizarse en los dos niveles de madurez inferiores, el nivel de $\mathrm{Ad}$ Hoc y Limitado, y el Formalizado y Predecible.
Lo anterior es relevante en la medida que como lo plantean Leskovar-Spacapan y Bastic (2007), los dos grandes enfoques, el estratégico y el tecnológico, donde se puede fundamentar la $\mathrm{Cl}$ en la organización, tienden a modelar la forma como el proceso de innovación se apropia en el quehacer empresarial; según lo planteado por los autores mencionados, se hace evidente que el enfoque estratégico prima en este caso particular para el área clave Proceso de Innovación, dado que en ésta área clave donde las interrelaciones de las personas al interior de la organización y de estas con el entorno, como un hecho cultural propio de la cotidianidad del trabajo, permiten capitalizar ideas y conocimientos para convertirlas en productos o servicios, procesos y sistemas que benefician la organización (Camisón y Villar, 2014; Liu y Jiang, 2016).

Dos elementos importantes se pueden observar dados los resultados obtenidos en el área clave Soporte Organizacional, primero, una relativa coincidencia en la forma como las cinco empresas responden a las variables y las ubican en los tres niveles de la escala de madurez, lo segundo, la alta dispersión entre los resultados, localizándolos en los extremos de la escala de madurez. Esta situación contrasta con el hecho de que la innovación exitosa es el resultado de la consolidación de procesos rigurosos y que con una determinada estructura, necesariamente responde a unas condiciones organizacionales que en el tiempo ayudan a garantizar un alto nivel de aciertos innovadores (Lema et al., 2005), situación que según la evidencia empírica, dista de lo observado en las empresas analizadas.

Es probable que el pragmatismo propio de las organizaciones empresariales, las cuales propenden por innovaciones que rápida y eficientemente se conviertan en productos o servicios, o incluso en mejoras para procesos y sistemas de gestión al interior de la organización, no conciban la utilidad de gestionar de una forma estructurada, metódica, amplia y con proyección en el tiempo el proceso de innovación, y por esta razón se explique el por qué en la escala de madurez hay diferencias tan marcadas entre unas variables y otras, o mucho más, no se encuentre aliciente a consolidar un superior nivel de madurez dado que las prácticas utilizadas proporcionan los resultados en innovación que la organización considera adecuados para su quehacer empresarial.

\section{Conclusiones}

Las variables de las $\mathrm{Cl}$ que llaman la atención por estar ubicadas en la escala de madurez Integración, Sinergia y Autonomía en las cinco empresas que se estudiaron son: Identificación de puntos de decisión claves en el 


\begin{tabular}{|c|c|c|c|}
\hline CI del área clave soporte organizacional & $\begin{array}{l}\text { Ad hoc y } \\
\text { limitado }\end{array}$ & $\begin{array}{c}\text { Formalizado y } \\
\text { predecible }\end{array}$ & $\begin{array}{c}\text { Integración, } \\
\text { sinergia y } \\
\text { autonomía }\end{array}$ \\
\hline \multicolumn{4}{|c|}{ 28. Formulación y comunicación de la estrategia de innovación. } \\
\hline \multicolumn{4}{|l|}{ 29. Valores y políticas organizacionales. } \\
\hline \multicolumn{4}{|l|}{ 30. Prácticas y procedimientos organizacionales. } \\
\hline \multicolumn{4}{|l|}{ 31. Fomento y apoyo al comportamiento innovador. } \\
\hline \multicolumn{4}{|l|}{ 32. Inversión en Innovación. } \\
\hline \multicolumn{4}{|l|}{ 33. Disponibilidad de recursos para la innovación. } \\
\hline \multicolumn{4}{|c|}{ 34. Infraestructura, sistemas y herramientas de apoyo a la innovación. } \\
\hline \multicolumn{4}{|l|}{ 35. Estructura organizacional e infraestructura instalada. } \\
\hline \multicolumn{4}{|l|}{ 36. Conformación de equipos de innovación. } \\
\hline \multicolumn{4}{|l|}{ 37. Comunicación y flujo de la información. } \\
\hline \multicolumn{4}{|l|}{ 38. Motivación de los individuos y equipos. } \\
\hline \multicolumn{4}{|l|}{ 39. Contratación de nuevas personas y asignación de roles. } \\
\hline \multicolumn{4}{|l|}{ 40. Medición de la innovación. } \\
\hline \multicolumn{4}{|l|}{ 41. Implementación y mejora del modelo de innovación. } \\
\hline 42. Implementación y mejora del modelo de innovación. & & & \\
\hline
\end{tabular}

\begin{tabular}{|ll|}
\hline & Empresa Sector Cárnico \\
& Empresa Sector Cerámico \\
$\ldots$ & Empresa Sector Electro-Gasodomésticos \\
$\ldots$ & Empresa Sector Concentrados para Animales \\
Fuente: Elaboración propia.
\end{tabular}

Figura 3. Madurez del área clave soporte organizacional por empresas.

proceso de los proyectos de innovación; Valores y políticas organizacionales; Conformación de equipos de innovación. Así mismo es importante reseñar que las variables localizadas en la escala Ad Hoc y Limitado son: Definición de la política de propiedad intelectual; Prácticas y procedimientos organizacionales; Estructura organizacional e infraestructura instalada;
Comunicación y flujo de la información; Contratación de nuevas personas y asignación de roles; Implementación y mejora del modelo de innovación.

En términos generales la empresa que se desempeña en el sector Cárnico es la que ha desarrollado un nivel de madurez superior a las demás empresas que parti- 
ciparon en el estudio; también se observa que la empresa del sector de Concentrados para Animales es la que tiene una escala de madurez inferior en el estudio.

El nivel de consolidación del proceso de innovación observado en las empresas objeto del estudio evidencia la dificultad para el afianzamiento de la madurez de las $\mathrm{Cl}$; cabría preguntar si el nivel actual en cuanto a la construcción de dichas capacidades tiene directa relación con procesos que han sido concebidos y diseñados a nivel estratégico pero que han tenido dificultad para ser desarrollados y operacionalizados, situación que es evidenciada en la poca madurez en la implementación y mejora del proceso de innovación y que se profundiza con variables fundamentales aún poco desarrollas o que no se han adecuado a las necesidades de una empresa que desea innovar como lo son: la estructura organizacional, prácticas y procesos organizacionales, los recursos disponibles para la investigación, la comunicación y el flujo de información, la contratación de personal.

No obstante la buena tendencia en la madurez que se observa en las variables de la $\mathrm{Cl}$ del Área Clave Proceso de Innovación ha permitido que las empresas estudiadas capitalicen diferentes iniciativas innovadoras. Lo anterior permite a las empresas garantizar en el corto plazo la posibilidad de desarrollos que coadyuvan los resultados de la gestión, pero esta tendencia podría convertirse en un riesgo a largo plazo puesto que las necesidades cortoplacistas marginalizarían las iniciativas tendientes a consolidar las variables de $\mathrm{Cl}$ de las áreas claves Conocimiento y Competencia; y Soporte Organizacional; queda pendiente para futuras investigaciones analizar las razones por las cuales se ha dificultado en las empresas incrementar los niveles de madurez en éstas áreas claves.

\section{Agradecimientos}

Este artículo es producto del proyecto de investigación "Madurez de la Capacidad de Innovación de las Empresas Antioqueñas líderes en Innovación, financiado por el Centro de Investigaciones y Consultoría y el Departamento de Ciencias Administrativas, adscritos a la Facultad de Ciencias Económicas de la Universidad de Antioquia, Medellín, Colombia; el cual se desarrolló entre el 2011 y el 2012.

\section{Referencias}

Aguirre J. 2010. Metodología para medir y evaluar las capacidades tecnológicas de innovación aplicando sistemas de lógica difusa: caso fábricas de software. Tesis de maestría no publicada. Medellín: Universidad Nacional de Colombia, Facultad de Minas.
Botero P, Castaño J. 2011. Una estructura de interrelación: Comité Universidad, Empresa, Estado en Antioquia, Estudio de Caso. 2007 [En línea] [Consultado el 28 de octubre de 2011] URL: http://www.udea.edu.co/portal/page/portal/BibliotecaProgramas/GestionTecnologica/ElemtosDiseno/Archivos/ComiteUEE/EstudioCasoUee/Anexo\%201.1\%20Est $\% 20 \mathrm{de} \% 20$ caso $\% 20$ del $\% 20$ CUEE.pdf.

Camisón C, Villar-López A. Organizational innovation as an enabler of technological innovation capabilities and firm performance. J Bus Res, 2014;67(1):2891-2902.

Essmann H, Toward. 2009. Innovation Capability Maturity. Tesis de doctorado no publicada. Stellenbosch: Stellenbosch University, Department of Industrial Engineering.

Essmann H, Du Preez N. An Innovation Capability Maturity Model: Development and initial application. World Academy of Science, Engineering and Technology. 2009;53(1): 435-446.

Hernández R, Fernández C, Baptista L. 2004. Metodología de la investigación. México: Mc Graw Hill.

Hurley R, Hult T. Innovation, Market Orientation, and Organizational Learning: An Integration and Empirical Examination. Journal of Marketing. 1998;62(3):42-54.

Lawson B, Samson D. Developing innovation capability in organisations: a dynamic capabilities approach. International Journal of Innovation Management. 2011;5(3):377-400.

Lema A. 2002. Elementos de Estadística Multivariada. Colombia: Silvano Ltda.

Lema R, Quadros R, Schmitz H. Reorganising global value chains and building innovation capabilities in Brazil and India. Res POlicy. 2015;44(7):1376-1386.

Liu L, Jiang Z. Influence of technological innovation capabilities on product competitiveness. Ind Manage Data Syst. 2016;116(5):883-902.

Leskovar-Spacapan G, Bastic M. Differences in organizations innovation capability in transition economy: Internal aspect of organizations strategic orientation. Technovation. 2007;27(9):533-546.

López C. 2009. Desarrollo de una de metodología de evaluación de capacidades para gestión de innovación. Tesis de maestría no publicada. Medellín: Universidad Nacional de Colombia, Facultad de Minas.

Jung $\mathrm{P}$, Lin B, Lin C. The construction and application of knowledge navigator model (KNM(TM)): An evaluation of knowledge management maturity. Expert Systems with Applications, 2009;36(2):4087-4100.

Robledo J, López C, Zapata W, Pérez JD. Desarrollo de una Metodología de Evaluación de Capacidades de Innovación. Perfil de Coyuntura Económica. 2010;1(15):133-148.

Yang C, Marlow P, Lu C. Assessing resources, logistics service capabilities, innovation capabilities and the performance of container shipping service in Taiwan. Int J Prod Econ. 2009;122(1):4-20. 